\title{
Cyclosporine A Regulates Pro-Inflammatory Cytokine Production in Ulcerative Colitis
}

\author{
Stefanie Steiner • Carolin Daniel • Anika Fischer • Imke Atreya • \\ Simon Hirschmann - Maximilian Waldner · Helmut Neumann • \\ Markus Neurath $\cdot$ Raja Atreya $\cdot$ Benno Weigmann
}

Received: 22 November 2013 / Accepted: 23 May 2014/Published online: 26 August 2014

(C) L. Hirszfeld Institute of Immunology and Experimental Therapy, Wroclaw, Poland 2014

\begin{abstract}
Crohn's disease (CD) and ulcerative colitis (UC) are the two major forms of inflammatory bowel diseases (IBD), which are defined as relapsing inflammations of the gastrointestinal tract. Cyclosporine A (CsA) is a potential rescue treatment to avoid colectomy in severe steroid-refractory UC patients. The molecular mechanism of action of CsA in UC is nevertheless still not well understood. The aim of this study was to investigate the effect of CsA on a possible modulation of cytokine production by peripheral blood mononuclear cells (PBMCs) of controls and patients with UC or CD. Upon CsA treatment, analyses of cytokine levels revealed a significant reduction of IL-13 expression in PBMCs from patients with UC, whereas other cytokine expression levels remained unaffected. To address the question whether CsA treatment impinges on the induction of cell death, apoptosis assays were performed using $\mathrm{CD}^{+}{ }^{+} \mathrm{T}$ cells from peripheral blood of patients suffering from either UC or CD. It became clear that CsA treatment resulted in a specific induction of apoptosis in samples from controls and patients with UC
\end{abstract}

R. Atreya and B. Weigmann contributed equally.

Electronic supplementary material The online version of this article (doi:10.1007/s00005-014-0309-7) contains supplementary material, which is available to authorized users.

S. Steiner - A. Fischer - I. Atreya - S. Hirschmann - M. Waldner ·

H. Neumann $\cdot$ M. Neurath $\cdot$ R. Atreya $\cdot$ B. Weigmann $(\square)$

Medical Clinic 1, Kussmaul Research Campus,

Friedrich-Alexander University of Erlangen-Nuremberg,

Hartmannstr. 14, 91052 Erlangen, Germany

e-mail: benno.weigmann@uk-erlangen.de

C. Daniel

Institute of Diabetes Research, Helmholtz Zentrum München, German Research Center for Environmental Health $(\mathrm{GmbH})$, Munich, Germany but not with $\mathrm{CD}$. Apoptosis induction was not mediated via the mitochondrial apoptosis pathway. The present data support the concept that CsA treatment modulates proinflammatory cytokine production and $\mathrm{T}$ cell survival in $\mathrm{UC}$ via the induction of apoptosis and might therefore help to explain the clinical efficacy of CsA in patients with UC.

Keywords IBD - Apoptosis - Cyclosporine A . Ulcerative colitis $\cdot$ IL-13

\section{Introduction}

Crohn's disease (CD) and ulcerative colitis (UC), the major forms of inflammatory bowel diseases (IBD), are chronic, relapsing, immunologically mediated disorders of the gastrointestinal tract. Although the aetiology and pathogenesis of IBD are far from being understood in detail, there is the general consensus that defects in the maintenance of immune responses, diminished barrier integrity, luminal antigens and alterations in pattern recognition receptors expressed in epithelial cells exacerbate the pathogenesis of IBD (Günther et al. 2011; Mandai et al. 2013; Vivinus-Nebot et al. 2014; Zheng et al. 2013). Another putative form of IBD is pouchitis, which is mostly developed by UC patients after ileal pouch-anal anastomosis (Coffey et al. 2009; Shen 2013). It has been shown before that IBD patients are characterized by altered profiles of pro- and anti-inflammatory cytokines in lamina propria CD4 ${ }^{+} \mathrm{T}$ cells (Desreumaux et al. 1997; Fuss et al. 1996). Particularly, they show an increase in the production of the pro-inflammatory cytokine tumor necrosis factor (TNF)- $\alpha$ as well as of the anti-inflammatory cytokine interleukin (IL)-10 (MacDonald and Monteleone 2001; Melgar et al. 2003). Additionally, cytokines of the IL-17 family were found to be important in IBD as levels of IL-17 
were increased in UC and CD patients (Fujino et al. 2003). Transforming growth factor (TGF)- $\beta$ is considered to be an inhibitory cytokine that plays a crucial role in regulating immunological homeostasis and inflammatory responses. Reduced TGF- $\beta$ activity is linked with the development of IBD (Marek et al. 2002). The anti-inflammatory cytokine IL4 has known immunosuppressive effects in the intestine, but both UC and CD patients do not show any alterations in IL-4 production (Camoglio et al. 1998; Fuss et al. 1996; SanchezMunoz et al. 2008). CD patients additionally revealed an increased amount of interferon (IFN)- $\gamma$ in lamina propria mononuclear cells (LPMCs) (Matsuoka et al. 2004). In contrast, an increased production of IL-5 and IL-13 of LPMCs is typically found in UC patients, who can be treated successfully with cyclosporine A (CsA) (Fuss et al. 1996; Heller et al. 2005). CsA is a cyclic peptide which is produced as a metabolite by the fungus Tolypocladium inflatum and can be used as an immunosuppressive agent to prevent rejection after organ transplantation as well as for the treatment of chronic inflammatory diseases (Kountouras et al. 2004; Meijssen 1998). It also inhibits the production of IL-2 by activated $T$ lymphocytes through a calcineurin-dependent pathway (Pino-Lagos et al. 2010; Siegel and Sands 2005). UC patients with severe steroid-refractory disease are often treated with CsA as a rescue medication to avoid an otherwise necessary colectomy. In this setting CsA is highly effective for short-term clinical improvement with immediate response rates of up to $80 \%$ (D'Haens et al. 2001; Lichtiger et al. 1994; Van Assche et al. 2003). The efficacy of CsA for the treatment of CD patients is discussed controversially. On the one hand CsA did not show any clinical efficacy in different randomized controlled trials with $\mathrm{CD}$ patients (Feagan et al. 1994; Stange et al. 1995). On the other hand, Brynskov et al. (1991) claimed that there is an initial beneficial effect of CsA on active $\mathrm{CD}$, but the benefit is not durable over a longer period of time. Currently, it is still unknown how CsA exerts its therapeutic effect in patients with UC on a molecular level and reasons are lacking to explain the absence of its efficacy in patients with CD. At present time, there are various treatment options like the antiTNF antibodies adalimumab and infliximab or the immunosuppressive drug azathioprine, which are clinically efficacious in the therapy of both disease entities. Infliximab is also used like CsA to treat steroid-refractory UC patients. It was shown, that Infliximab and CsA are comparable concerning rates of colectomy after 3 and 12 months, adverse drug reactions and postoperative complications (Chang et al. 2013). Based on the assumption that IBD is caused by an uncontrolled activation and enhanced resistance to apoptosis of lamina propria $\mathrm{T}$ lymphocytes, it has been demonstrated that the central mechanism of action of these substances is attributed to their capacity to induce apoptosis in mucosal $\mathrm{T}$ cells, thereby inhibiting the intestinal inflammatory process (Atreya et al. 2011; ten Hove et al. 2002; Tiede et al. 2003; Van den Brande et al. 2007). Apoptosis induced by death receptors is suggested to be an important mechanism for the downregulation of local immune responses (Doering et al. 2004). After binding of a specific ligand to its death receptor, a death-induced signaling complex (DISC) is built, consisting of Fas-associated death-domain containing protein and pro-caspase 8 (Neurath et al. 2001). DISC is responsible for inducing the autoproteolytic cleavage of pro-caspase 8 , which leads to the activation of caspase 8 and its release into the cytosol (Lee et al. 2012). Subsequently, the caspase signaling cascade in the cytosol is activated. The ultimate activation of caspase 3 can occur via a mitochondrial dependent or independent pathway. Low amounts of caspase 8 induce apoptosis in a mitochondrial dependent manner mediated by cleavage of the pro-apoptotic BID molecule which leads to the release of cytochrome $\mathrm{c}$ from the mitochondria into the cytosol (Neurath et al. 2001). In this scenario the expression of the antiapoptotic proteins $\mathrm{Bcl}-2$ and $\mathrm{Bcl}-\mathrm{xL}$ as well as $\mathrm{Bax}$ is a reliable indicator for the activation of this pathway (ten Hove et al. 2002). High amounts of caspase 8 directly activate caspase 3 thereby promoting the induction of apoptosis (Neurath et al. 2001).

In the present study we focused on the impact of CsA to modulate cytokine expression levels of peripheral blood mononuclear cells (PBMCs) from healthy controls as well as patients with UC or CD upon stimulation in vitro. Therefore, we hypothesized that the effect of CsA treatment is associated with the regulation of $\mathrm{T}$ cell apoptosis. Our results indicated that CsA could have a protective role in the treatment of UC by inducing T cell apoptosis with a consequent downregulation of pro-inflammatory cytokines.

\section{Materials and methods}

\section{Patients}

Venous blood was obtained from CD $(n=22)$, UC $(n=18)$ and control patients $(n=13)$. The median age of the patients' cohort was 35 (18-72). Patients with different subtypes of UC and CD were included in this study (Table 1). The IBD patients were treated with 5-Aminosalizylates, corticosteroids, azathioprine or the anti-TNF antibodies infliximab and adalimumab, when the blood was taken. All IBD patients were naïve to calcineurin inhibitor treatment (CsA or Tacrolimus) at the time of the study (Table 2). CD patients had a mean Harvey-Bradshaw Index (HBI) score of 7.5 and UC patients a mean partial Mayo score of 4.8. PBMCs were subsequently isolated from the venous blood and used for further analyses. The use of human blood was approved by the local ethical committee. 
Table 1 Subtypes of UC and CD patients included in the study

\begin{tabular}{ll}
\hline Subtype & $(\%)$ \\
\hline UC & \\
Pancolitis & 72.20 \\
Left-sided & 16.70 \\
Proctitis & 11.10 \\
CD & \\
Colitis & \\
Ileocolitis & 40.9 \\
Inflammatory & 63.6 \\
Stricturing & 36.4 \\
Penetrating & 36.4 \\
\hline
\end{tabular}

The Table shows the UC and CD subtypes of the patients included in the study. The percentage of patients belonging to each type is indicated

Table 2 Pre-treatment at the time of blood withdrawal of UC and CD patients

\begin{tabular}{llc}
\hline & UC $(\%)$ & CD (\%) \\
\hline $\begin{array}{l}\text { Response to previous or current therapy } \\
\text { Steroids }\end{array}$ & \\
$\quad$ Responder & 52.9 & 70.0 \\
$\quad$ Non-responder & 47.1 & 30.0 \\
& & \\
Azathioprine & 0 & 10.5 \\
$\quad$ Responder & 100 & 89.5 \\
$\quad$ Non-responder & & \\
Anti-TNF-Ab & 46.7 & 93.8 \\
Responder & 53.3 & 6.3 \\
Non-responder &
\end{tabular}

The Table summarizes the response of the patients included in the study to previous or current therapy. Patients were classified into three groups regarding medical treatment which include therapy with steroids, azathioprine and anti-TNF antibodies. The percentage of patients in regard to their response to each therapy is indicated

All patients gave written informed consent to participate in this study.

\section{Isolation of PBMCs}

PBMCs were isolated from whole blood of patients and controls using human Pancoll reagent (PAN Biotech $\mathrm{GmbH}$, Germany) for density gradient separation. PBMCs $\left(5 \times 10^{6}\right.$ cells per well) were cultured in RPMI medium 1,640 (Gibco, USA) containing $10 \%$ FCS (PAN Biotech GmbH, Germany), $1 \%$ L-Glutamine (200 mM; Gibco, USA) and $1 \%$ Penicillin/Streptomycin (PAA, UK) for $48 \mathrm{~h}$ in the presence of $10 \mu \mathrm{g} / \mathrm{ml} \mathrm{CsA}$ (Sandimmun,
Novartis Pharma, Germany) or medium alone. Additionally, all cells were stimulated with anti-human CD3 (clone OKT3; BioLegend, USA) and anti-human CD28 antibodies (clone CD28.2; BioLegend, USA). Supernatants were taken for ELISA assays. The cells were washed with PBS and taken for FACS-analysis, RNA isolation and immunofluorescent stainings.

To determine the influence of IL-2 on cytokine production cells were isolated and stimulated as described above. PBMCs were additionally incubated with IL-2 (100 U/ml; ImmunoTools, Germany).

\section{ELISA}

IL-13, IL-17A, IL-17F, IL-23, TGF- $\beta$, IFN- $\gamma$ and TNF- $\alpha$ levels were determined by Ready-SET-Go ELISA sets (eBioscience, USA) and IL-4, IL-5 and IL-10 levels were determined by ELISA MAX ${ }^{\mathrm{TM}}$ Deluxe Set (BioLegend, USA) according to the manufacturer's instructions. Supernatants of PBMCs were taken after $48 \mathrm{~h}$ of incubation and diluted in $1 \times$ Assay Diluent to determine cytokine levels. For the determination of IL-13 levels, supernatants were discarded after $48 \mathrm{~h}$, new medium was added and cells were again stimulated with anti-CD3 and anti-CD28 antibodies. After $24 \mathrm{~h}$ supernatants were taken and diluted 1:2 in $1 \times$ Assay Diluent for ELISA assay. Standards were diluted according to manufacturer's instructions.

\section{Flow Cytometry}

The induction of apoptosis in T cells was analyzed by flow cytometry. Approximately 100.000 cells were incubated for $20 \mathrm{~min}$ at $4{ }^{\circ} \mathrm{C}$ with an allophycocyanin (APC) antihuman CD4 antibody (1:200; BioLegend, USA). After washing cells were stained for Annexin V (Annexin V-FITC Apoptosis Detection Kit I; BD Pharmingen, USA) according to manufacturer's instructions. Fluorescence was acquired and analyzed with a LSR Fortessa Cytometer (BD Bioscience, USA), using BD FACSDiva Software. Dead cells were excluded using 7AAD staining (7AAD Viability Staining Solution; eBioscience, USA). For the investigation of the cellular characteristics of the isolated PBMCs, cells were stained with APC anti-human CD3 (1:100; TONBO bioscience, USA), VioBlue anti-human CD4 (1:50; Miltenyi, Germany) and Pe/Cy7 anti-human CD8a (1:200; BioLegend, USA).

Inhibition of Caspase 8

The PBMCs of healthy controls were cultured for $48 \mathrm{~h}$ with RPMI medium 1,640 (Gibco, USA) containing $10 \%$ FCS (PAN Biotech GmbH, Germany), $1 \%$ L-Glutamine (200 mM; Gibco, USA) and $1 \%$ Penicillin/Streptomycin 
Table 3 Qiagen primers used for qPCR analysis

\begin{tabular}{ll}
\hline Primer & Order number \\
\hline Caspase 8 & QT00052416 \\
Bcl-2 & QT00025011 \\
Bcl-xL & QT00236712 \\
Bax & QT00031192 \\
IL-13 & QT00000511 \\
18sRNA & QT00199367
\end{tabular}

All primers used in this study were ordered from Qiagen (Germany). The Table lists all primers with their order number

(PAA, UK) in the presence or absence of CsA $(10 \mu \mathrm{g} / \mathrm{ml}$, Sandimmun, Novartis Pharma). zVad (1:1000; Bachem, Switzerland) and Necrostatin-1 (1:1000; Sigma-Aldrich, USA) were added additionally separately from each other or together. Cells were stained with an APC anti-human CD4 antibody (1:200; BioLegend, USA) as described above, and with propidium iodide (BD Pharmingen, USA) as well as with Pacific Blue Annexin V (1:50; BioLegend, USA) and for activated caspase 8 using CaspGLOW Fluorescein Active Caspase 8 Staining Kit (eBioscience, USA). Cells were then analyzed as described above.

\section{Quantitative Real-Time PCR}

After washing, 3,000,000 PBMCs were lysed in $350 \mu \mathrm{l}$ lysis buffer (Macherey-Nagel, Germany) and stored at $-20{ }^{\circ} \mathrm{C}$. Total RNA was isolated using Nucleo Spin RNA II kit (Macherey-Nagel, Germany). The isolation was performed according to manufacturer's instructions. Quality of RNA samples was determined by spectrophotometrical measurements. RNA was reversed transcribed to cDNA using iScript cDNA Synthesis Kit (BioRAD, USA). cDNA was diluted 1:10 for further qPCR analyses. Quantitative real-time PCR was performed with $2 \times$ SensiFAST SYBR No-ROX Mix (Bioline, UK) and specific primers for caspase 8, Bcl-2, Bcl-xL, Bax and IL-13. All primers were ordered from Qiagen (Germany), see Table 3. Levels of 18s RNA were used to normalize gene expression levels of the other genes. Bcl-2/Bax ratio was calculated.

\section{Immunofluorescent Staining}

Cells were taken for immunofluorescent staining and analyzed by microscopy. Staining of CD4 was done using an anti-human CD4 antibody (clone: RPA-T4; eBioscience, USA) at a concentration of 1:50. Goat anti-mouse Cy3 (1:200; ImmunoResearch Laboratories Inc., UK) was chosen as secondary antibody. TUNEL staining (DeadEnd Fluorometric TUNEL System; Promega, USA) was done according to manufacturer's instructions. Nuclei of cells were counterstained with Hoechst dye 33342 (Invitrogen, USA).

\section{Results}

The immunosuppressive drug CsA can be successfully used in treating UC patients. In contrast, for CD patients this therapy does not lead to an improvement of the clinical symptoms. The molecular mechanism of CsA action is not well understood, so we asked the question whether CsA treatment can modulate cytokine production and apoptosis induction.

\section{CsA Altered Cytokine Expression in PBMCs of UC Patients}

In this study, supernatants of PBMCs of control and IBD patients cultured with or without CsA were analyzed for the levels of different cytokines. Composition of the isolated cells was determined by FACS analysis and revealed that there is no marked alteration between the investigated groups. Most of the isolated cells were $\mathrm{CD}^{+} \mathrm{CD}^{+}$(Supplementary Fig. 1). It was found that the production of the pro-inflammatory cytokine TNF- $\alpha$ as well as IL-17A and IL-10 was significantly reduced after CsA treatment in PBMCs of all patients groups. Production of IL-17F was significantly diminished by addition of CsA in controls and CD patients, but UC patients did not show a significant alteration. IL-5 concentration in supernatants was significantly decreased in controls and UC patients whereas concentration did not change in UC patients after CsA treatment. Investigation of TGF- $\beta$ production revealed comparable cytokine levels for UC and CD patients upon application of CsA and a significant reduction in controls. Additionally, IL-23 production was investigated, but cytokine levels were too low to be detected (data not shown). Levels of the Th1-related cytokine IFN- $\gamma$, which plays a crucial role in the pathogenesis of $\mathrm{CD}$, remained unaffected after the addition of $10 \mu \mathrm{g} / \mathrm{ml} \mathrm{CsA}$. The same could be shown for IL-4 production. In UC patients, levels of IL-13 were significantly reduced after CsA treatment in vitro. Importantly in PBMCs of controls and CD patients IL-13 production was unaffected by CsA treatment (Fig. 1a). IL-13 production in controls was independent of IL-2, as there was no difference in IL-13 levels after addition of CsA, which is known to inhibit IL-2 production (Supplementary Fig. 2). The relative expression of IL-13 mRNA also showed significant differences after addition of CsA in UC patients, whereas expression remained unaffected in controls and CD patients (Fig. 1b). These experiments revealed significantly reduced levels of IL-13 
protein production after CsA treatment, which was exclusively visible in PBMCs of UC patients.

\section{CsA Treatment Induced Apoptosis in PBMCs of UC Patients and Controls}

To investigate whether the altered cytokine production is due to an induction of apoptosis, we treated PBMCs of healthy controls, UC and CD patients with CsA for $48 \mathrm{~h}$ followed by Annexin $\mathrm{V}$ staining. In this setting, necrotic $7 \mathrm{AAD}^{+}$cells were excluded and only $7 \mathrm{AAD}^{-} \mathrm{CD} 4^{+}$cells were included for analysis of apoptosis (Fig. 2a, left diagram). The number of viable and apoptotic $\mathrm{CD} 4^{+}$cells was significantly increased upon application of CsA in PBMCs of control and UC patients, while there was no significant induction of apoptosis in CD patients (Fig. 2a, right diagram). To confirm these findings stainings for CD4 and TUNEL, as a marker for apoptotic cells, were performed on cultured PBMCs. The untreated $\mathrm{CD}^{+}$ peripheral blood cells were nearly all negative for TUNEL-staining in all three patient groups. $\mathrm{CD} 4^{+}$PBMCs of control patients showed an increased number of TUNEL positive cells after CsA treatment. In accordance with the data of the flow cytometric experiments, there was a marked induction of apoptosis in $\mathrm{CD}^{+} \mathrm{T}$ cells in UC patients, whereas nearly none of the TUNEL positive cells in CD patients were CD4 positive (Fig. 2b). Thus, we were able to show, that cultured $\mathrm{CD}^{+}$peripheral blood cells of controls and UC patients underwent apoptosis after CsA treatment, while this effect was not visible in cells of CD patients.

\section{Apoptosis of PBMCs after CsA Treatment Occurred in a Mitochondrial Independent Way}

Apoptosis can be induced either by a mitochondrialdependent or -independent way. Therefore we analyzed which pathway mediates CsA induced apoptosis. qPCR analysis with cDNA of untreated and CsA treated PBMCs of all patients groups revealed that the relative expression of pro-apoptotic caspase 8 was significantly increased in all investigated groups upon addition of CsA. The relative expression of the anti-apoptotic factor Bcl-xL, which is important for the mitochondrial dependent pathway of apoptosis, remained unaffected by CsA treatment (Fig. 3a). The Bcl-2/Bax ratio showed no significant differences between untreated and CsA treated samples of controls, UC and $\mathrm{CD}$ patients (Fig. 3b). These results indicate, that apoptosis was activated independently of the mitochondrial pathway. We further analyzed if caspase 8 is necessary for inducing CsA mediated apoptosis. Therefore we cultured PBMCs of healthy controls with and without CsA. Additionally, zVad, an inhibitor for activated caspase 8 , or
Necrostatin-1, an inhibitor of necrosis, were added separately from each other or together. We could show that $\mathrm{CD} 4^{+}$caspase $8^{+}$cells revealed a diminished induction of apoptosis after inhibition of activated caspase 8 , whereas $\mathrm{CD} 4^{+}$caspase $8^{-} \mathrm{T}$ cells were not influenced regarding their rate of apoptosis by $\mathrm{CsA}, \mathrm{zVad}$ or Necrostatin- 1 application (Fig. 3c). This experiment revealed that CsA mediated apoptosis of PBMCs was dependent on activated caspase 8 signaling.

All results showed no correlation to the different medical treatments of patients at the time of blood withdrawal and their response to previous or current therapy (Supplementary Fig. 4).

\section{Discussion}

In this study we assessed the effects of CsA treatment on the production of cytokines and on the induction of apoptosis in peripheral blood $\mathrm{T}$ cells from healthy controls and patients suffering from IBD.

It is known that CsA treatment can modulate mRNA expression of cytokines as well as the corresponding protein levels (Bunikowski et al. 2001; Harness et al. 2001; Rafiq et al. 2000). We could show that there is an alteration in the cytokine production of PBMCs after CsA treatment in UC patients. Expression of the pro-inflammatory cytokine TNF- $\alpha$ is increased in both, UC and CD patients (Hering et al. 2012). Our results revealed that levels of TNF- $\alpha$ were significantly reduced in all groups after CsA treatment. IL-4 is known to play an important role in IBD (Sanchez-Munoz et al. 2008). In connection with CsA IL-4 production is reduced in different in vitro studies (Andersson et al. 1992; Tsuda et al. 2012). We could show, that in the case of IBD patients and controls, IL-4 levels are reduced in all groups investigated. Additionally, our results revealed a significant reduction of IL5 production after CsA treatment in controls and UC patients. This effect was also described for other in vitro studies, in which blood cells of healthy donors were treated with CsA (Andersson et al. 1992). Members of the IL-17 family of cytokines were described to show elevated levels in IBD patients with active disease (SanchezMunoz et al. 2008). IL-17A production was significantly reduced after CsA treatment in all groups, whereas IL$17 \mathrm{~F}$ levels were significantly reduced in controls and CD patients, but not in UC patients. Decreased IL-17 levels were also described in a study, in which the influence of CsA on IL-17 production of PBMCs of patients suffering from Behcets disease was investigated (Chi et al. 2010). Expression of the cytokine IL-10 was shown to be increased in UC patients with active disease (Melgar et al. 2003). Our results revealed that IL-10 levels were 
significantly decreased by addition of CsA in all groups investigated. Because TGF- $\beta$ is known to have a different expression pattern in CD and UC patients, we examined whether CsA treatment influences TGF- $\beta$ production differently in PBMCs of IBD patients (Del Zotto et al. 2003). TGF- $\beta$ levels were significantly reduced in controls by addition of CsA, but did not show any differences in UC and CD patients. Based on these findings we concluded that CsA mediated differences of these cytokines in UC patients is not decisive for an efficient therapy of UC patients with CsA, as this effect was also visible in $\mathrm{CD}$ patients. Because $\mathrm{CD}$ is discussed to be a Th1-like disorder, whereas UC is more characterized as a Th2-like response, we investigated the levels of IFN- $\gamma$ and IL-13 (Kountouras et al. 2004). The production of IFN- $\gamma$, a classical Th1 cytokine, did not show a significant difference between untreated and CsA treated blood cells of controls, UC and CD patients. The production of IL13, a Th2 cytokine, was significantly reduced after addition of CsA only in PBMCs of UC patients, whereas control patients and $\mathrm{CD}$ patients showed a slight increase in IL-13 production. Similar results were illustrated in a study, in which children, suffering from severe atopic dermatitis, were treated with a low-dose CsA microemulsion and afterwards cytokine levels of IFN- $\gamma$ and IL13 were investigated. The authors could also show that IFN- $\gamma$ production was unaffected by CsA treatment, whereas IL-13 levels were decreased after addition of CsA. They concluded that low-doses of CsA prevent activation of Th2 cells (Bunikowski et al. 2001). It is known that calcineurin inhibitors, like CsA, reduce IL-2 levels (Granelli-Piperno 1988). Our results revealed that the reduction of IL-13 in UC patients was independent of IL-2 reduction by the calcineurin inhibition. Additionally, IL-13 is proposed to play an important role in fistulizing CD. IL-13 expression is increased in transitional cells at the border of fistula tracks, where it induces expression of target genes involved in fistula formation (Mannon and Reinisch 2012). In our study we investigated cells isolated of whole blood samples, so that we could exclude any influence of IL-13 producing cells involved in fistulizing $\mathrm{CD}$. We assume from our results that the effective treatment of UC patients by CsA could at least partially be mediated by a specific reduction of IL-13 cytokine expression levels.

The decrease in IL-13 production can be explained by the CsA mediated induction of apoptosis. This process plays a crucial role in $\mathrm{T}$ cell development and homeostasis (Shale et al. 2013). In IBD, LPMCs of patients show a decreased susceptibility to apoptosis which leads to an uncontrolled expansion of effector $\mathrm{T}$ cells causing gut inflammation (Boirivant et al. 1996; Mudter and Neurath 2007). We could
Fig. 1 Significant decrease in IL-13 levels in UC patients after CsA treatment. a Cytokine levels were determined by ELISA. TNF- $\alpha$, IL$17 \mathrm{~A}$ and IL-10 levels were significantly reduced in all groups after CsA treatment, whereas IFN- $\gamma$ levels remained unaffected after CsA treatment in all groups. IL-17F concentration was significantly diminished upon addition of CsA in controls and CD patients. Levels of IL-5 showed a significant reduction in controls and UC patients after CsA treatment, but not in CD patients. The concentration of TGF- $\beta$ was decreased significantly in controls, but showed no alteration in UC and CD patients. IL-4 levels were not affected by $\mathrm{CsA}$ treatment in all investigated groups. Addition of CsA resulted only in a significant reduction of IL-13 levels in colitis patients. Concentrations of IL-13 did not show a significant difference in controls and CD patients. b Real-time PCR analysis showed that the relative expression of IL-13 was reduced significantly by CsA treatment in UC patients whereas expression was unaffected after addition of CsA in controls and CD patients. Significant differences are indicated with asterisks $* p<0.05, * * p<0.01, * * * p<0.001$

observe an increase in the number of $\mathrm{CD} 4^{+} \mathrm{T}$ cells undergoing apoptosis in UC patients whereas apoptosis induction in $\mathrm{CD}$ patients was unaffected by CsA treatment. We could demonstrate this effect by Annexin V staining, which is a specific marker for apoptosis (Demchenko 2012). Additionally, we performed immunofluorescent staining for TUNEL, which is known to detect DNA fragmentation by labeling double strand brakes of apoptotic cells. It was shown that there is a clear discrimination between apoptotic and necrotic cells in the TUNEL assay, as the number of double strand breaks can be correlated with the fluorescent intensity of the TUNEL assay (Wlodkowic et al. 2011). Additionally, we could show a direct influence of CsA on cytokine production, e.g. IL-13 and IFN$\gamma$, in T cells from control patients even when apoptosis is inhibited (Supplementary Fig. 3). The induction of apoptosis is believed to play a central role in the molecular mechanism of action of clinically efficacious therapies in IBD (Van den Brande et al. 2007). The finding that CsA treatment can induce apoptosis of $\mathrm{T}$ cells was also shown in a model for experimental autoimmune encephalomyelitis (EAE) (McCombe et al. 1999). In this study, CsA treated Lewis rats suffering from EAE prevented the development of disease during treatment, but after ceasing CsA treatment the rats developed delayed signs of disease. Single-dose injection of CsA revealed an induction of apoptosis in $\mathrm{T}$ cells.

Intracellular proteases of the caspase family are crucial for the induction of apoptosis. Caspases can be activated by two signaling routes, the extrinsic and the intrinsic pathway (Becker et al. 2013). For the last-mentioned the antiapoptotic proteins $\mathrm{Bcl}-\mathrm{xL}$ and $\mathrm{Bcl}-2$ as well as the proapoptotic protein Bax, all members of the Bcl-2 family, play a pivotal role (Gross et al. 1999). In our study, we could show that there is no alteration in the expression of Bcl-2 family member proteins with CsA treatment, 

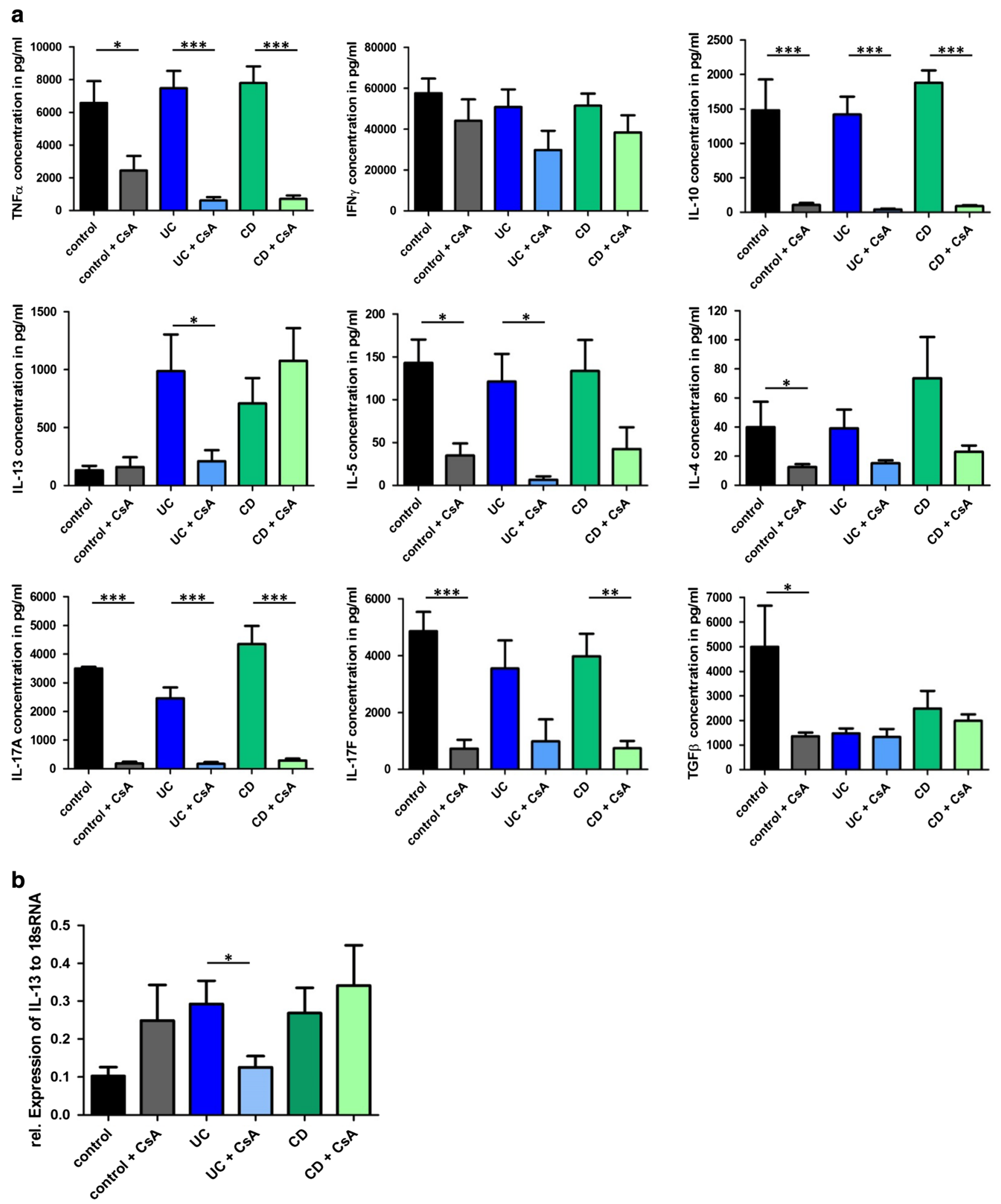
Fig. 2 Apoptosis induction after CsA treatment in $\mathrm{CD}^{+}{ }^{+} \mathrm{T}$ cells. a Human PBMCs of colitis and CD patients as well as healthy controls were stained for CD4, 7AAD and Annexin V and analyzed by FACS.

Majority of these cells were alive, as analyzed by 7AAD staining (left diagram). In the right diagram it is shown, that the number of $\mathrm{CD}^{+}$Annexin $\mathrm{V}^{+}$cells was significantly increased after CsA treatment in controls and colitis patients. CD patients showed no significant difference. For this experiment 10 controls, 11 colitis and $9 \mathrm{CD}$ patients were used. b PBMCs were double stained for CD4 and TUNEL. Cells of controls and UC patients revealed an increased number of double positive cells after CsA treatment, while blood cells of CD patients were not positive for both markers. Significant differences are indicated with asterisks $* p<0.05$,

$* * p<0.01, * * * p<0.001$
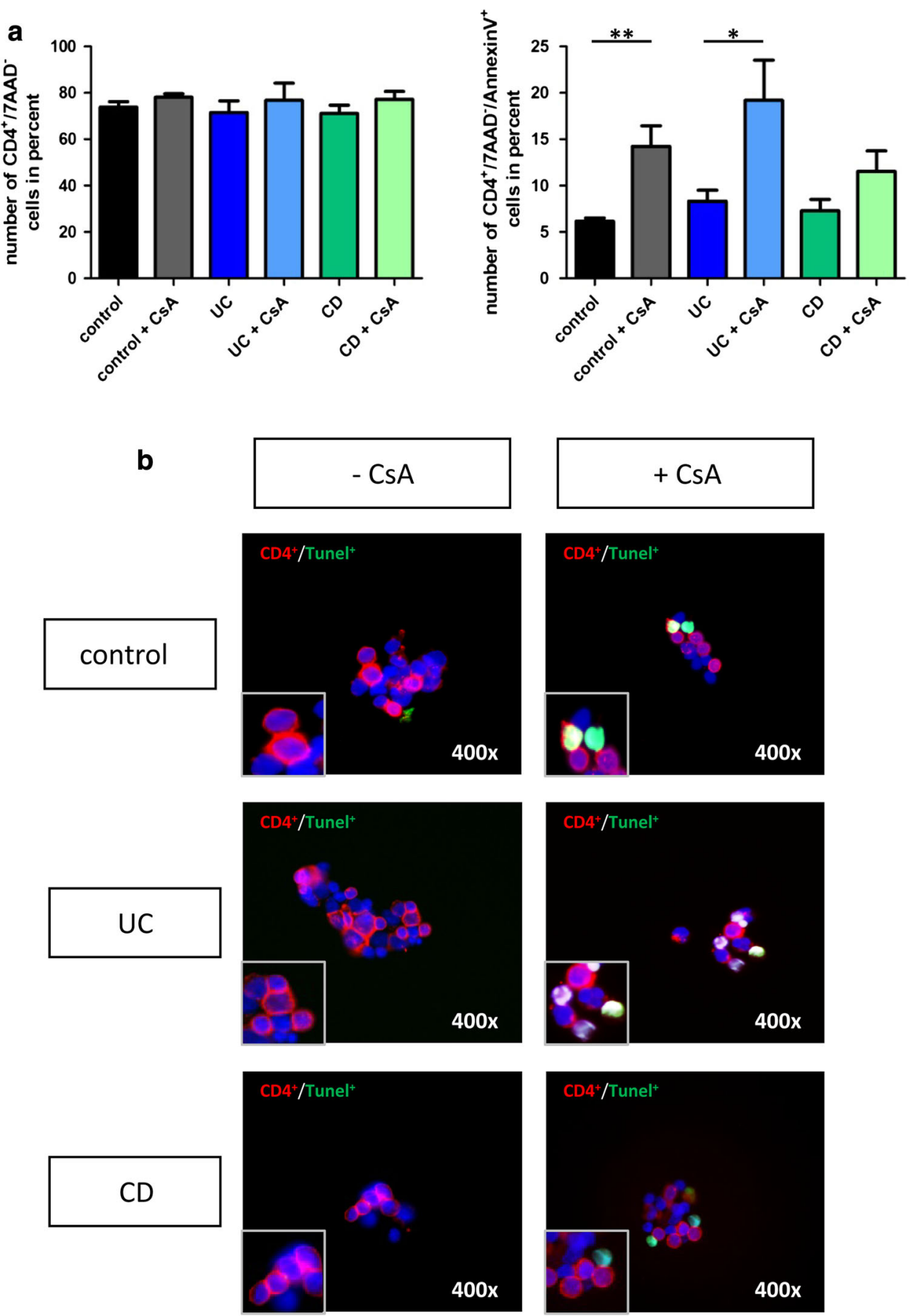

especially in UC patients. The extrinsic apoptosis pathway is dependent on the expression of activated caspase 8 (Becker et al. 2013). Because of the alteration in caspase 8 expression depending on CsA treatment, we supposed that CsA mediated apoptosis requires activated caspase 8 , which is necessary for triggering apoptotic cell death via death receptor activation and the caspase cascade (Günther et al. 2011). By specific inhibition of caspase 8 with $\mathrm{zVad}$ in PBMCs of controls we could show that the number of
$\mathrm{CD}^{+} \mathrm{T}$ cells undergoing apoptosis was reduced independently of CsA treatment. It was shown that human LPMCs can undergo apoptosis via induction of either the extrinsic or the intrinsic pathway in vitro (Boirivant et al. 1996; Hoffmann et al. 2007). Thus, we conclude that CsA induces apoptosis of $\mathrm{CD}^{+} \mathrm{T}$ cells via the extrinsic pathway.

IBD patients, suffering from Th2 associated UC, can be treated successfully with CsA. Our study demonstrates for the first time that CsA selectively induces apoptosis in 
a
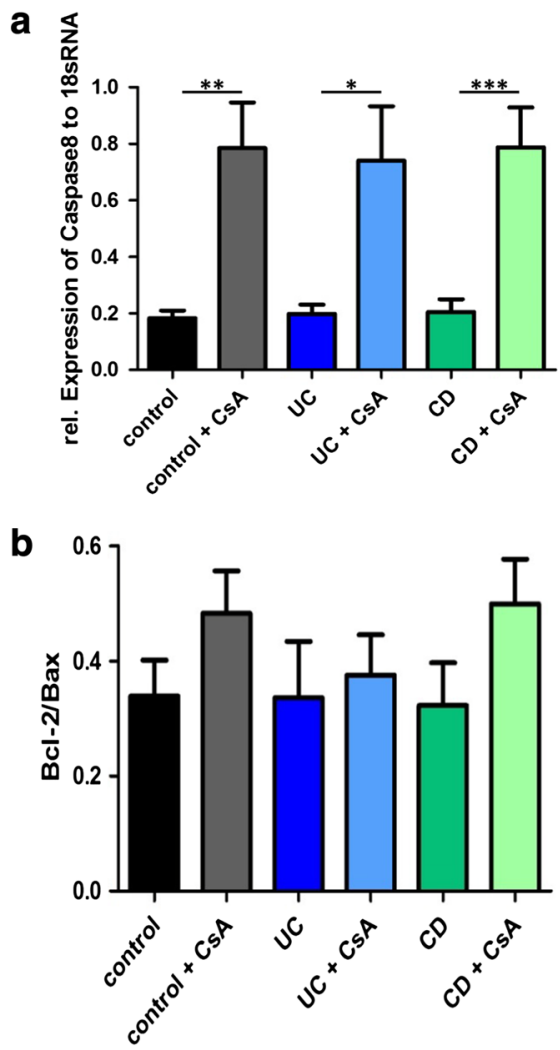

C +

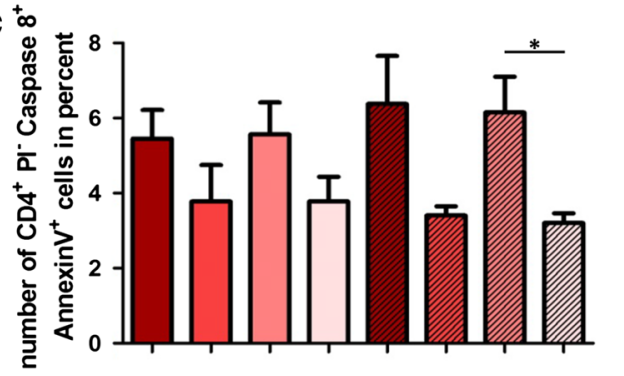

$\mathrm{CD} 3 / \mathrm{CD} 28$

CsA

zVad

Nec-1

Fig. 3 CsA mediated induction of apoptosis in a mitochondrial independent way. a Real-time PCR analysis revealed a significantly increased expression of the pro-apoptotic caspase 8 after treatment of PBMCs with CsA in all patients groups. For this analysis 10 controls, $8 \mathrm{UC}$ patients and $9 \mathrm{CD}$ patients were used. The relative expression of the anti-apoptotic Bcl-xL was unaffected by CsA treatment. 10 controls, $9 \mathrm{UC}$ patients and $10 \mathrm{CD}$ patients were analyzed for altered $\mathrm{Bcl}-\mathrm{xL}$ expression. b Relative expression levels of Bcl-2 and Bax were determined by real-time PCR analysis and ratio of Bcl-2 and Bax was calculated. No significant differences could be detected

$\mathrm{CD} 4^{+}$blood cells of UC patients in a mitochondrial independent way associated with a diminished production of the Th2-like cytokine IL-13.
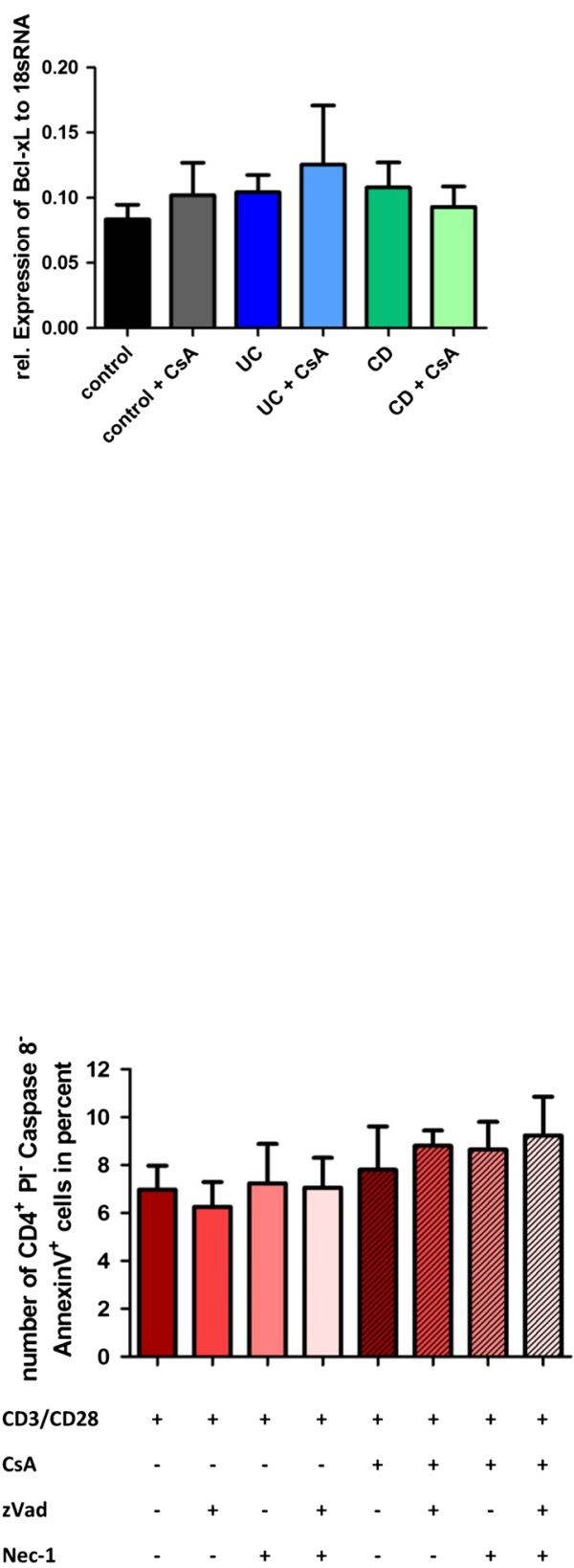

between the untreated and CsA treated group of controls, UC and CD patients. c Isolated PBMCs of four healthy controls were cultured with and without CsA, zVad and Necrostatin-1. These cells were stained for CD4, propidium iodide, activated caspase 8 and Annexin $\mathrm{V}$ and analyzed by flow cytometry. With inhibition of activated caspase 8 there was a decrease in the number of apoptotic $\mathrm{CD}^{+} \mathrm{T}$ cells independent of CsA and Necrostatin-1 treatment (left diagram). Cells, negative for activated caspase 8, induced apoptosis independent of CsA and zVad (right diagram). Significant differences are indicated with asterisks $* p<0.05, * * p<0.01, * * * p<0.001$

Acknowledgments This research was supported by the German Research Foundation (clinical research unit KFO257) and ELAN and the Emerging Fields Initiative of the University Erlangen-Nuremberg. Funds from the German Ministry for Science and Education (BMBF 
ZooMAP 01KI1003G) were used for sample preparation. We thank A. von Berg and L. Sologub for excellent technical assistance.

\section{References}

Andersson J, Nagy S, Groth CG et al (1992) Effects of FK506 and cyclosporin A on cytokine production studied in vitro at a singlecell level. Immunology 75:136-142

Atreya R, Zimmer M, Bartsch B et al (2011) Antibodies against tumor necrosis factor (TNF) induce T-cell apoptosis in patients with inflammatory bowel diseases via TNF receptor 2 and intestinal $\mathrm{CD} 4^{+}$macrophages. Gastroenterology 141:2026-2038

Becker C, Watson AJ, Neurath MF (2013) Complex roles of caspases in the pathogenesis of inflammatory bowel disease. Gastroenterology 144:283-293

Boirivant M, Pica R, DeMaria R et al (1996) Stimulated human lamina propria $\mathrm{T}$ cells manifest enhanced Fas-mediated apoptosis. J Clin Invest 98:2616-2622

Brynskov J, Freund L, Norby Rasmussen S et al (1991) Final report on a placebo-controlled, double-blind, randomized, multicentre trial of cyclosporin treatment in active chronic Crohn's disease. Scand J Gastroenterol 26:689-695

Bunikowski R, Staab D, Kussebi F et al (2001) Low-dose cyclosporin A microemulsion in children with severe atopic dermatitis: clinical and immunological effects. Pediatr Allergy Immunol 12:216-223

Camoglio L, Te Velde AA, Tigges AJ et al (1998) Altered expression of interferon-gamma and interleukin- 4 in inflammatory bowel disease. Inflamm Bowel Dis 4:285-290

Chang KH, Burke JP, Coffey JC (2013) Infliximab versus cyclosporine as rescue therapy in acute severe steroid-refractory ulcerative colitis: a systematic review and meta-analysis. Int J Colorectal Dis 28:287-293

Chi W, Yang P, Zhu X et al (2010) Production of interleukin-17 in Behcet's disease is inhibited by cyclosporin A. Mol Vis $16: 880-886$

Coffey JC, Rowan F, Burke J et al (2009) Pathogenesis of and unifying hypothesis for idiopathic pouchitis. Am J Gastroenterol 104:1013-1023

Del Zotto B, Mumolo G, Pronio AM et al (2003) TGF-beta1 production in inflammatory bowel disease: differing production patterns in Crohn's disease and ulcerative colitis. Clin Exp Immunol 134:120-126

Demchenko AP (2012) The change of cellular membranes on apoptosis: fluorescence detection. Exp Oncol 34:263-268

Desreumaux P, Brandt E, Gambiez L et al (1997) Distinct cytokine patterns in early and chronic ileal lesions of Crohn's disease. Gastroenterology 113:118-126

D'Haens G, Lemmens L, Geboes K et al (2001) Intravenous cyclosporine versus intravenous corticosteroids as single therapy for severe attacks of ulcerative colitis. Gastroenterology 120:1323-1329

Doering J, Begue B, Lentze MJ et al (2004) Induction of $\mathrm{T}$ lymphocyte apoptosis by sulphasalazine in patients with Crohn's disease. Gut 53:1632-1638

Feagan BG, McDonald JW, Rochon J et al (1994) Low-dose cyclosporine for the treatment of Crohn's disease. The canadian Crohns relapse prevention trial investigators. $\mathrm{N}$ Engl J Med 330:1846-1851

Fujino S, Andoh A, Bamba S et al (2003) Increased expression of interleukin 17 in inflammatory bowel disease. Gut 52:65-70

Fuss IJ, Neurath M, Boirivant M et al (1996) Disparate CD4+ lamina propria (LP) lymphokine secretion profiles in inflammatory bowel disease. Crohn's disease LP cells manifest increased secretion of IFN-gamma, whereas ulcerative colitis LP cells manifest increased secretion of IL-5. J Immunol 157:1261-1270

Granelli-Piperno A (1988) In situ hybridization for interleukin 2 and interleukin 2 receptor mRNA in T cells activated in the presence or absence of cyclosporin A. J Exp Med 168:1649-1658

Gross A, McDonnell JM, Korsmeyer SJ (1999) BCL-2 family members and the mitochondria in apoptosis. Genes Dev 13:1899-1911

Günther C, Martini E, Wittkopf N et al (2011) Caspase-8 regulates TNF-alpha-induced epithelial necroptosis and terminal ileitis. Nature 477:335-339

Harness J, Pender MP, McCombe PA (2001) Cyclosporin A treatment modulates cytokine mRNA expression by inflammatory cells extracted from the spinal cord of rats with experimental autoimmune encephalomyelitis induced by inoculation with myelin basic protein. J Neurol Sci 187:7-16

Heller F, Florian P, Bojarski C et al (2005) Interleukin-13 is the key effector Th2 cytokine in ulcerative colitis that affects epithelial tight junctions, apoptosis, and cell restitution. Gastroenterology 129:550-564

Hering NA, Fromm M, Schulzke JD (2012) Determinants of colonic barrier function in inflammatory bowel disease and potential therapeutics. J Physiol 590(Pt 5):1035-1044

Hoffmann U, Heilmann K, Hayford C et al (2007) CD44v7 ligation downregulates the inflammatory immune response in Crohn's disease patients by apoptosis induction in mononuclear cells from the lamina propria. Cell Death Differ 14:1542-1551

Kountouras J, Zavos C, Chatzopoulos D (2004) Immunomodulatory benefits of cyclosporine A in inflammatory bowel disease. J Cell Mol Med 8:317-328

Lee EW, Seo J, Jeong M et al (2012) The roles of FADD in extrinsic apoptosis and necroptosis. BMB Rep 45:496-508

Lichtiger S, Present DH, Kornbluth A et al (1994) Cyclosporine in severe ulcerative colitis refractory to steroid therapy. N Engl J Med 330:1841-1845

MacDonald TT, Monteleone G (2001) IL-12 and Th1 immune responses in human Peyer's patches. Trends Immunol 22:244-247

Mandai Y, Takahashi D, Hase K et al (2013) Distinct roles for CXCR6 and CXCR6 CD4 T cells in the pathogenesis of chronic colitis. PLoS ONE 8:e65488

Mannon P, Reinisch W (2012) Interleukin 13 and its role in gut defence and inflammation. Gut 61:1765-1773

Marek A, Brodzicki J, Liberek A et al (2002) TGF-beta (transforming growth factor-beta) in chronic inflammatory conditions-a new diagnostic and prognostic marker? Med Sci Monit 8:145-151

Matsuoka K, Inoue N, Sato T et al (2004) T-bet upregulation and subsequent interleukin 12 stimulation are essential for induction of Th1 mediated immunopathology in Crohn's disease. Gut 53:1303-1308

McCombe PA, Harness J, Pender MP (1999) Effects of cyclosporin A treatment on clinical course and inflammatory cell apoptosis in experimental autoimmune encephalomyelitis induced in Lewis rats by inoculation with myelin basic protein. J Neuroimmunol 97:60-69

Meijssen MA (1998) Cyclosporine and inflammatory bowel disease: buying time. Mediat Inflamm 7:145-147

Melgar S, Yeung MM, Bas A et al (2003) Over-expression of interleukin 10 in mucosal $\mathrm{T}$ cells of patients with active ulcerative colitis. Clin Exp Immunol 134:127-137

Mudter J, Neurath MF (2007) Apoptosis of T cells and the control of inflammatory bowel disease: therapeutic implications. Gut $56: 293-303$

Neurath MF, Finotto S, Fuss I et al (2001) Regulation of T-cell apoptosis in inflammatory bowel disease: to die or not to die, that is the mucosal question. Trends Immunol 22:21-26 
Pino-Lagos K, Michea P, Sauma D et al (2010) Cyclosporin A-treated dendritic cells may affect the outcome of organ transplantation by decreasing CD4 + CD25 + regulatory $\mathrm{T}$ cell proliferation. Biol Res 43:333-337

Rafiq K, Bullens DM, Kasran A et al (2000) Differences in regulatory pathways identify subgroups of $\mathrm{T}$ cell-derived Th2 cytokines. Clin Exp Immunol 121:86-93

Sanchez-Munoz F, Dominguez-Lopez A, Yamamoto-Furusho JK (2008) Role of cytokines in inflammatory bowel disease. World J Gastroenterol 14:4280-4288

Shale M, Schiering C, Powrie F (2013) $\mathrm{CD}^{+}$T-cell subsets in intestinal inflammation. Immunol Rev 252:164-182

Shen B (2013) Pouchitis: what every gastroenterologist needs to know. Clin Gastroenterol Hepatol 11:1538-1549

Siegel CA, Sands BE (2005) Review article: practical management of inflammatory bowel disease patients taking immunomodulators. Aliment Pharmacol Ther 22:1-16

Stange EF, Modigliani R, Pena AS et al (1995) European trial of cyclosporine in chronic active Crohn's disease: a 12-month study. The European Study Group. Gastroenterology 109:774-782

ten Hove T, van Montfrans C, Peppelenbosch MP et al (2002) Infliximab treatment induces apoptosis of lamina propria $\mathrm{T}$ lymphocytes in Crohn's disease. Gut 50:206-211

Tiede I, Fritz G, Strand S et al (2003) CD28-dependent Rac1 activation is the molecular target of azathioprine in primary human CD4+ T lymphocytes. J Clin Invest 111:1133-1145
Tsuda K, Yamanaka K, Kitagawa H et al (2012) Calcineurin inhibitors suppress cytokine production from memory $\mathrm{T}$ cells and differentiation of naive $\mathrm{T}$ cells into cytokine-producing mature T cells. PLoS ONE 7:e31465

Van Assche G, D'Haens G, Noman M et al (2003) Randomized, double-blind comparison of $4 \mathrm{mg} / \mathrm{kg}$ versus $2 \mathrm{mg} / \mathrm{kg}$ intravenous cyclosporine in severe ulcerative colitis. Gastroenterology 125:1025-1031

Van den Brande JM, Koehler TC, Zelinkova Z et al (2007) Prediction of antitumour necrosis factor clinical efficacy by real-time visualisation of apoptosis in patients with Crohn's disease. Gut 56:509-517

Vivinus-Nebot M, Frin-Mathy G, Bzioueche H et al (2014) Functional bowel symptoms in quiescent inflammatory bowel diseases: role of epithelial barrier disruption and low-grade inflammation. Gut 63:744-752

Wlodkowic D, Telford W, Skommer J et al (2011) Apoptosis and beyond: cytometry in studies of programmed cell death. Methods Cell Biol 103:55-98

Zheng B, Morgan ME, van de Kant HJ et al (2013) Transcriptional modulation of pattern recognition receptors in acute colitis in mice. Biochim Biophys Acta 1832:2162-2172 